\title{
DURABILIDAD NATURAL Y ADQUIRIDA DE 27 MADERAS TROPICALES EN CONDICION DE CAMPO
}

Leticia Guevara Salnicov *

David LLuncor Mendoza **

\section{RESUMEN}

En el Proyecto "Estudios Básicos y Tecnológicos de Maderas" que ejecuta el Convenio INIA-IIAPP se ha determinado la durabilidad natural y adquirida por tratamiento preservador de dieciséis y trece maderas de Loreto, respectivamente, y la durabilidad natural de once de Ucayali. Las probetas de madera destinadas a ser ensayadas con preservador se las sometió a inmersión prolongada de temperatura ambiente en una solución acuosa al $4 \%$ de sales cupro-cromo-bóricas, luego se las instaló en dos parcelas "cementerio" ubicadas en el bosque de shiringa de la Estación Experimental Forestal Pucallpa - INIA, según procedimiento propuesto por JUNAC (1981). Las evaluaciones y calificación del estado sanitario de las probetas se efectuó de acuerdo a normas de la American Wood Preservers Association (1985). Se efectuó tres evaluaciones. En la última, trece maderas de Loreto están completamente destruidas y las tres restantes presentan pudrición incipiente. De las maderas de Ucayali, nueve están completamente destruidas y con pudrición avanzada. En las preservadas se observa mayor resistencia al deterioro biológico, que se evidencia en menor velocidad de deterioro. Por ello, es recomendable que, en usos en contacto con el suelo, se utilice madera preservada. Se debe efectuar ensayos para determinar la eficacia de productos y procesos.

\section{INTRODUCCION}

La durabilidad natural de la madera puede definirse como la resistencia que opone a todo tipo de deterioro. Para fines prácticos y considerando la preponderancia del deterioro biológico, la durabilidad natural se refiere a la resistencia que opone la madera al ataque de hongos e insectos xilófagos.

Los factores responsables de la durabilidad natural son numerosos. Algunos dependen de las características intrínsecas de la madera, otros se relacionan con las circunstancias de uso. La invasión de agentes de deterioro muestra una sucesión natural en función del contenido de humedad de la madera.

* $\quad$ Ing $^{0}$ Forestal. Investigador IIAP

** M.Sc. Forestal. Investigador IIAP 
Para la determinación de la durabilidad natural de la madera se propone dos métodos. En laboratorio, bajo condiciones reguladas de temperatura, humedad relativa y disponibilidad de aire, se expone la madera al ataque de cultivos puros de hongos xilófagos y colonias de insectos. Los resultados son referenciales de un posible comportamiento de la madera en servicio. Las pruebas definitivas se llevan a acabo en parcelas "cementerio", donde se expone la madera a las condiciones del medio y ataque múltiple de organismos xilófagos.

Considerando que la durabilidad natural es una característica tecnológica indispensable para definir usos de la madera especialmente aquellos en que las condiciones de destrucción biológica son máximas, se plantea en el presente estudio determinar el grado de durabilidad natural de 27 maderas y durabilidad adquirida por tratamiento preservador de 13 maderas.

\section{REVISION DE LITERATURA}

\section{DURABILIDAD NATURAL DE LA MADERA}

Así se denomina, según Cartwrigt (1960) y Gonzales (1974) a la resistencia que opone al ataque de agentes biológicos de deterioro, tales como hongos e insectos xilófagos, perforadores marinos, y agentes no biológicos de deterioro, tales como desgaste mecánico, intemperismo atmosférico y al peligro del fuego. Para fines prácticos, la durabilidad puede considerarse como la resistencia que opone la madera a la pudrición.

La durabilidad es una propiedad de la madera en extremo variable. Varia entre las diferentes especies leñosas, entre los diferentes árboles de una misma especie, y aun dentro de un mismo árbol. Los factores responsables de la durabilidad de la madera son numerosos y variables; algunos dependen de las características y condiciones de la madera misma, otros están relacionados con las circunstancias que concurren en su uso. La gran durabilidad del duramen con respecto a la albura se atribuye a la presencia de algunas condiciones físicas y químicas que suceden en la formación del duramen. En efecto, Kollman (1959) sostienen que el duramen se protege de hongos e insectos lignícolas mediante obstrucción mecánica de los vasos, taponamiento de las punteaduras e impregnación de las paredes celulares, Además se depositan en las células del duramen sustancias polifenólicas, básicamente derivados del ácido shiquímico, cinámico y cafeico, cuyas combinaciones con compuestos orgánicos determinan la gran variedad de preservadores de la madera, capaces de ejercer efectos tóxicos y/o repelentes contra organismos lignícolas. 


\section{AGENTES BIOLÓGICOS DE DETERIORO}

A causa de su naturaleza orgánica, la madera, está expuesta a numerosos agentes de deterioro biológico. La frecuencia e intensidad de estos dependen de las condiciones en que se encuentre la madera. En todo caso, se establece una sucesión de agentes biológicos de deterioro en función del contenido de humedad. Esto es válido desde el árbol en pie hasta la madera en servicio.

\section{HONGOS LIGNÍCOLAS}

Los hongos lignícolas son los principales enemigos de la madera. Cartwrigh (1960) distingue particularmente a los hongos propiamente xilófagos, capaces de disolver enzimáticamente la pared de las células leñosas. Aunque la mayoría pertenecen a la clase Basidiomycetes, también pueden causarlo especies de la clase Ascomycetes, especialmente en maderas con alto contenido de humedad y/o escasa disponibilidad de oxígeno.

\section{INSECTOS LIGNÍCOLAS}

Existe una serie de insectos lignícolas que invaden la madera en busca de alimento o lugar de incubación. Económicamente importantes, tanto como por la frecuencia como por el grado de deterioro que ocasionan, son los termes. Son insectos sociales que constituyen colonias organizadas funcionalmente. Una vez invadida la madera, practican galerías internas, reduciendo la resistencia, hasta la total destrucción. Son frecuentes en zonas de clima cálido-húmedo. Se distinguen los termes subterráneos (Rhinotermitidae), no subterráneos o de madera húmeda (Kalotermitidae) y de madera seca (Termitidae).

\section{EVALUACIÓN EXPERIMENTAL DE LA DURABILIDAD NATURAL DE LA MADERA}

Con la finalidad de estudiar la resistencia de las maderas al ataque de organismos se efectúa ensayos de laboratorio y de campo con metodología normal izada.

En laboratorio se regula las condiciones a fin de favorecer al máximo el ataque (humedad, temperatura, sustrato y aire). Se mide el deterioro causado por agentes particularmente agresivos. Los procedimientos establecidos por la Asociación Americana de Preservadores de Madera-AWPA (1985) permiten llevar a cabo ensayos en periodos relativamente cortos, Se usan hongos xilófagos y especies 
de termes que se desarrollan bien en condiciones de laboratorio y son importantes destructores de madera en servicio. Los resultados permiten discriminar maderas de acuerdo al grado de durabilidad natural. Aquellas que muestran resistencia al deterioro son ensayadas en campo.

Las pruebas de campo se las realiza en áreas seleccionadas por las características climáticas (temperatura y humedad relativa), presencia de hongos xilófagos y termes subterráneos, vegetación y tipo de suelo. El período de exposición es variable. Se exige, como mínimo, dos anos. El máximo es indefinido, dependiendo del comportamiento de las maderas.

Los procedimientos normalizados recomiendan usar probetas de sección transversal pequeña, enterradas hasta la mitad de la longitud, y efectuar revisiones del estado sanitario con una periodicidad no mayor de un año. Los resultados permiten concluir sobre la durabilidad natural de la madera y, eventualmente, la toxicidad, resistencia a la lixiviación y permanencia de los preservadores en las maderas que han sido tratadas.

Las evaluaciones se analizan de acuerdo al sistema de clasificación propuesto por AWPA (1985) como se indica en el cuadro 1.

\section{MATERIALES Y MÉTODOS}

\section{LUGAR}

Los ensayos se efectuaron en la Estación Experimental Forestal Pucallpa-IN IA.

\section{MADERAS}

Los cuadros 2 y 3 muestran la relación de maderas ensayadas procedentes de la zona del río Yavarí-Colonia Angamos, Loreto y del Bosque Nacional Alexander von Humholdt, Ucayali.

\section{METODOLOGIA}

\section{Preparación de probetas}

Se preparó probetas de $5 \times 5 \times 25 \mathrm{~cm}$., secas al aire y cepilladas en caras y cantos. Las destinadas a ser ensayadas con preservador se las sumergió en una solución al $4 \%$ de sales cupro-cromo-bóricas a temperatura ambiente durante 14 días. 


\section{Instalación en el "cementerio"}

Las probetas se las distribuyó al azar en tres parcelas. Una de éstas, de 2,5 x 2,5 m, conformada por probetas de maderas preservadas de Loreto, otra, de 2,5 x $2,5 \mathrm{~m}$, con probetas de maderas sin preservar de Loreto; ambas instaladas en marzo de 1989, la última, de 1,5 x 2,0 m, con maderas sin preservar de Ucayali en setiembre de 1989 .

\section{Evaluación}

Se realizó tres inspecciones en las tres parcelas. La primera en diciembre de 1990, la segunda en noviembre de 1991 y la tercera en octubre de 1992, 21, 32 y 43 meses de instaladas las probetas de madera de Loreto y a 16, 27, y 38 meses de instaladas las probetas de maderas de Ucayali.

\section{RESULTADOS Y DISCUSION}

Bajo las condiciones de la prueba, las posibilidades de deterioro fueron máximas. Las condiciones ambientales: clima húmedo y cálido, alta precipitación y las evidencias de ataque múltiple de hongos e insectos lignícolas actuando simultáneamente favorecen el deterioro biológico y la lixiviación y/o detoxificación.

De igual forma, la pequeña sección transversal (aunque no es la más pequeña sección transversal recomendada por las normas técnicas) favorece la velocidad de pudrición y ataque de termes, hasta alcanzar el grado $\mathrm{O}$ (cero) en períodos relativamente cortos.

El ensayo con probetas cepilladas, requisito establecido por las especificaciones de la Junta de Acuerdo de Cartagena (1981), es inadecuado. En probetas cepilladas se reduce el área superficial real, la cual influye en la absorción de agua, en el contacto efectivo entre la madera y el suelo y en la infección por esporas fungosas. Por otra parte, si el procedimiento normalizado supone condiciones similares a las de servicio para piezas en contacto directo con el suelo, con o sin tratamiento preservador, tales como postes, durmientes, bases, fundación y estacas, no se requiere cepillado.

De las dieciséis maderas de Loreto ensayadas sin tratamiento preservador, cumala blanca (duramen/albura y albura) y huira caspi (albura y duramen) fueron destruidas en 21 meses, moena sin olor (duramen), caupuri colorado (albura y duramen), tangarana (duramen), manchan caspi (duramen), papelillo caspi (albura), shiringa (duramen), shiringa masha (duramen), ana caspi (albura/duramen), balata sapotina (duramen) y pucuna caspi (albura/duramen) fueron destruidas en 32 meses, machimango colorado (duramen), y machimango blanco (albura/duramen), están con 
síntomas de pudrición y/o termes, moena amarilla (albura/duramen) y quinilla colorada (duramen) muestran ligeros síntomas de pudrición.

De las once maderas de Ucayali ensayadas, todas las probetas fueron de duramen. La manchinga es destruida por pudrición en 16 meses; chimicua, aguano masha, pumaquiro, yutubanco, yacushapana, mashonaste, tamamuri, shihuahuaco, y estoraque son destruidas por pudrición (4), termes (1) o ambos (5) en 28 meses, quedando el tahuarí en estado avanzado de pudrición y ataque moderado de termes.

En la mayor parte de los casos no se ha advertido una diferencia sustancial en la resistencia de albura y duramen. También se ha observado que la frecuencia de la pudrición es superior en el ataque de termes. En la primera evaluación, de 24 probetas ensayadas, 18 fueron atacadas por pudrición, siete por termes y cuatro permanecen indemnes, En la segunda evaluación, de las siete probetas, todas estaban atacadas por pudrición y tres por termes, en la mayoría de los casos aislados, no se detectó pudrición exclusivamente causada por hongos Basidiomycetex. En todo caso, podrían haber estado simultáneamente con especies de Ascomycetes, los que, por su agresividad y tolerancia, pueden desarrollar bien aun en sustratos deficientes de aire por consecuencia de altos contenidos de humedad en la madera.

De las probetas ensayadas con tratamiento preservador, el deterioro biológico fue más lento pero se presentó en forma definida en la tercera evaluación. De las trece maderas, nueve fueron destruidos o estaban en avanzado estado de deterioro: tangarana, manchan caspi, machimango colorado, balata sapotina, cumala blanca, shiringa masha, caupuri colorado, shiringa; albura de machimango blanco, de quinilla colorada y de pucuna caspi. Quedaron indemnes las probetas de moena sin olor, albura/duramen de quinilla colorada, de machimango blanco y duramen de pucuna caspi.

$\mathrm{Si}$ es cierto que para fines prácticos el incremento de vida útil como consecuencia del tratamiento preservador no es realmente relevante, lo que merece resaltar es que aún a bajas retenciones (26\% de la retención mínima establecida por ITINTEC para usos en contacto directo con el suelo, $9,6 \mathrm{~kg} / \mathrm{m}^{3}$ ) y con agresivas condiciones ambientales, la preservación es una opción para el uso de estas maderas, asegurando períodos de vida útil compatibles con los costos adicionales por concepto de tratamiento. 


\section{CONCLUSIONES}

- De las dieciséis maderas de Loreto y once de Ucayali ensayadas en áreas "cementerio", dos de Loreto - moena amarilla y quinilla colorada - demuestran tener mayor durabilidad natural.

- La principal causa de destrucción es por pudrición mixta, causado por Basidiomycetes y Ascomycetes.

- La resistencia al deterioro biológico en trece maderas de Loreto preservadas por inmersión prolongada en una solución al $4 \%$ de sales cupro-cromo-bóricas a una retención promedio de $2,6 \mathrm{~kg} / \mathrm{m} 3$ es mayor que en madera sin preservar.

\section{Cuadro 1}

CLASIFICACIÓN DE MADERAS DE ACUERDO AL ESTADO SANITARIO

\begin{tabular}{cll}
\hline Grado & \multicolumn{2}{c}{ Condición de las probetas } \\
& Pudrición & Termes \\
\hline 10 & Libre & Libre \\
9 & Incipiente & Indicios \\
7 & Media & Ataque medio \\
4 & Avanzada & Ataque fuerte \\
0 & Falla & Fractura \\
\hline
\end{tabular}




\section{Cuadro 2}

\section{MADERAS DE LA ZONA DEL RÍO YAVARÍ - COLONIA ANGAMOS, IQUITOS}

\section{Nombre común}

Ana caspi

Balata sapotina

Cumala blanca

Caupuri colorado

Huira caspi

Machimango blanco

Machimango colorado

Manchari caspi

Moena amarilla

\section{LAURACEAE}

Moena sin olor

Papelillo caspi

Pucuna caspi

Quinilla colorada

Shiringa

Shiringa masha

Tangarana
Nombre científico

Apuleia molaris Spruce ex Benth

Chrisophvllum ucuquirana-blanca

(Aubreville \& Ppennington)

Osteophoeum platyspermun A.DC.Warb

Virola pavonis (A.D.C.) A.C.Smith

Tapirira quianensis Auhlet. Smith

Lecythis peruviana L.O.Williams

Escheweleira timbucchensi.s Knuth

Vantanea parviflora Larn.

Aniba puchury-minor (Martius) Mez.

Ruizteranis Trichantera (Spruce ex

Warm)

Cariniana decandra Ducke

Iryanthera tricornis Ducke

Puteria caimito Ruiz \& Pavon

Hevea guianensis Aublec

Micranda spruceana (Bail)R.E. Smith

Sclerolobium melinonei Harás
Familia

CAESALPINACEA

SAPOTACEAE

MYRISTICACEAE

MYRISTICACEAE

ANACARDIACEAE

LECYTHIDACEAE

LECYTHIDACEAE

HUMIRACEAE

VOCHISIACEAE

LECYTHIDACEAE

MYRISTICACEAE

SAPOTACEAE

EUPHORBIACEAE

EUPHORBIACEAE

CAESALPINACEAE 


\section{Cuadro 3}

\section{MADERAS DEL BOSQUE NACIONAL ALEXANDER VON HUMBOLDT, UCAYALI}

Nombre común

Aguano masha

Chimicua

Estoraque

Machinga

Machimango

Mashonaste

Pumaquiro

Shihuahuaco

Tahuarí

Yacushapana

Yutubanco
Nombre científico

Paramachaerium ormosiodea

Perebea aff mollis (P \& F) Rusby

Miroxylum blasamun

Brosimun alicastrum Sw. Spp boliviarensi MORACEAE

Schweilera ovalifolia (APDC) Ndz.

Clarisia racemosa

Aspidosperma macrocarpon

Dipterix adorata (Aubl.) Wild

Tabebuia serratofolia (Vahl) Nich

Terminalia amazónica (JF Gemí) Excell

BIGNONEACEAE

Drypetes amazonicus Sterr. Spp. peruviana
Familia

FABACEAE

MORACEAE

PAPILIONACEAE

LECYTHIDACEAE MORACEAE

APOCYNACEAE

PAPILONACEAE 


\section{Cuadro 4}

\section{DURABILIDAD NATURAL DE 16 MADERAS DE LORETO}

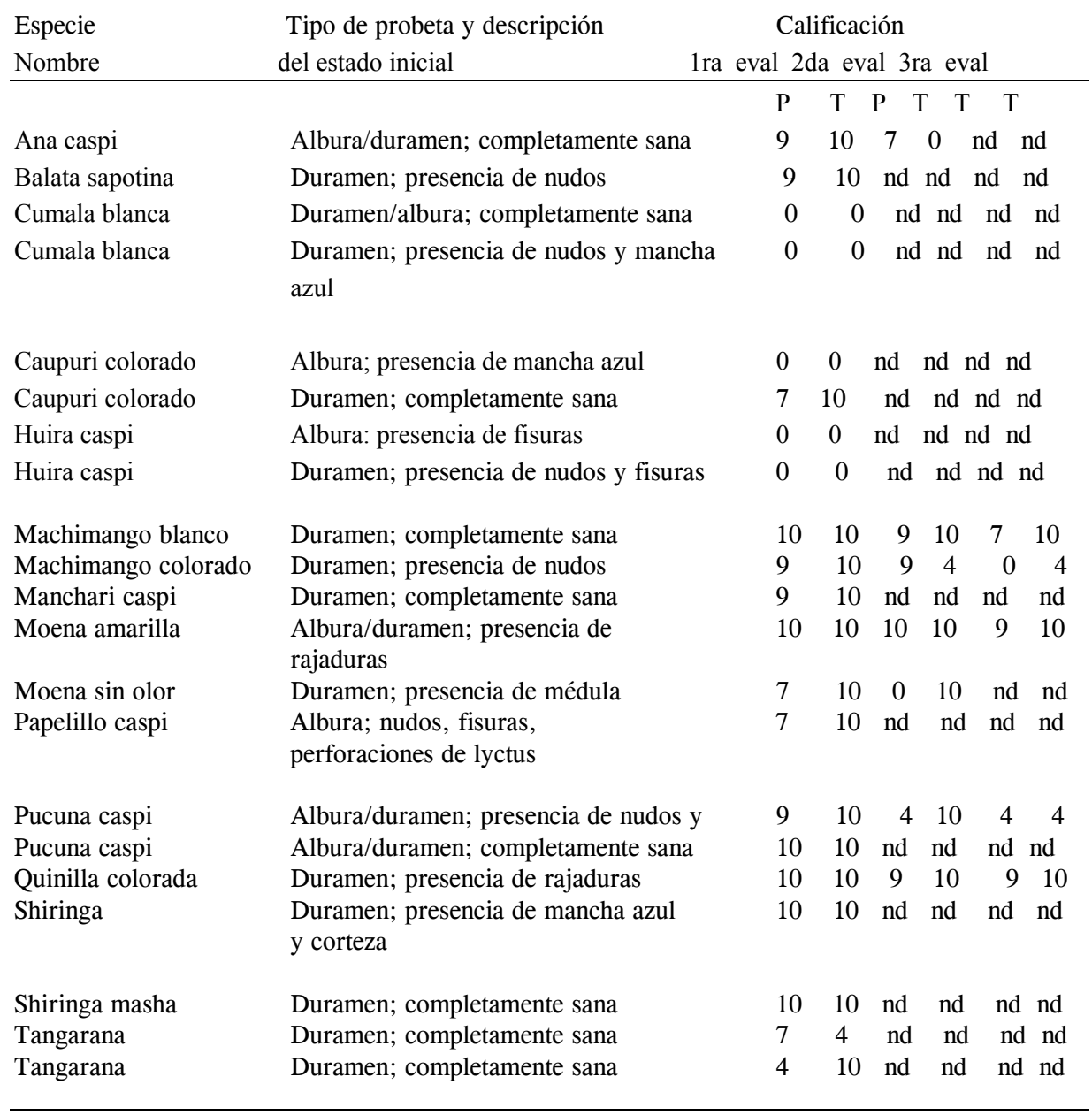




\section{Cuadro 5}

\section{DURABILIDAD NATURAL DE DIEZ MADERAS DE UCAYALI}

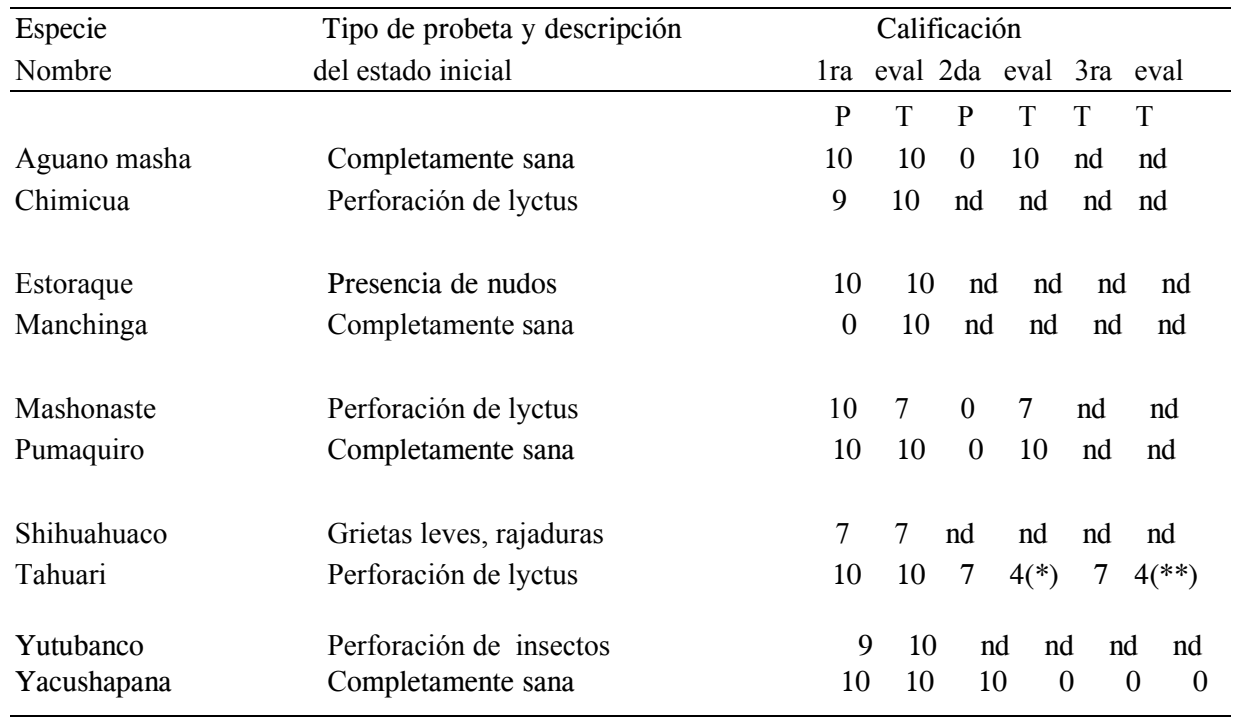




\section{Cuadro 6}

\section{DURABILIDAD ADQUIRIDA POR TRATAMIENTO PRESERVADOR DE TRECE MADERAS DE LORETO}

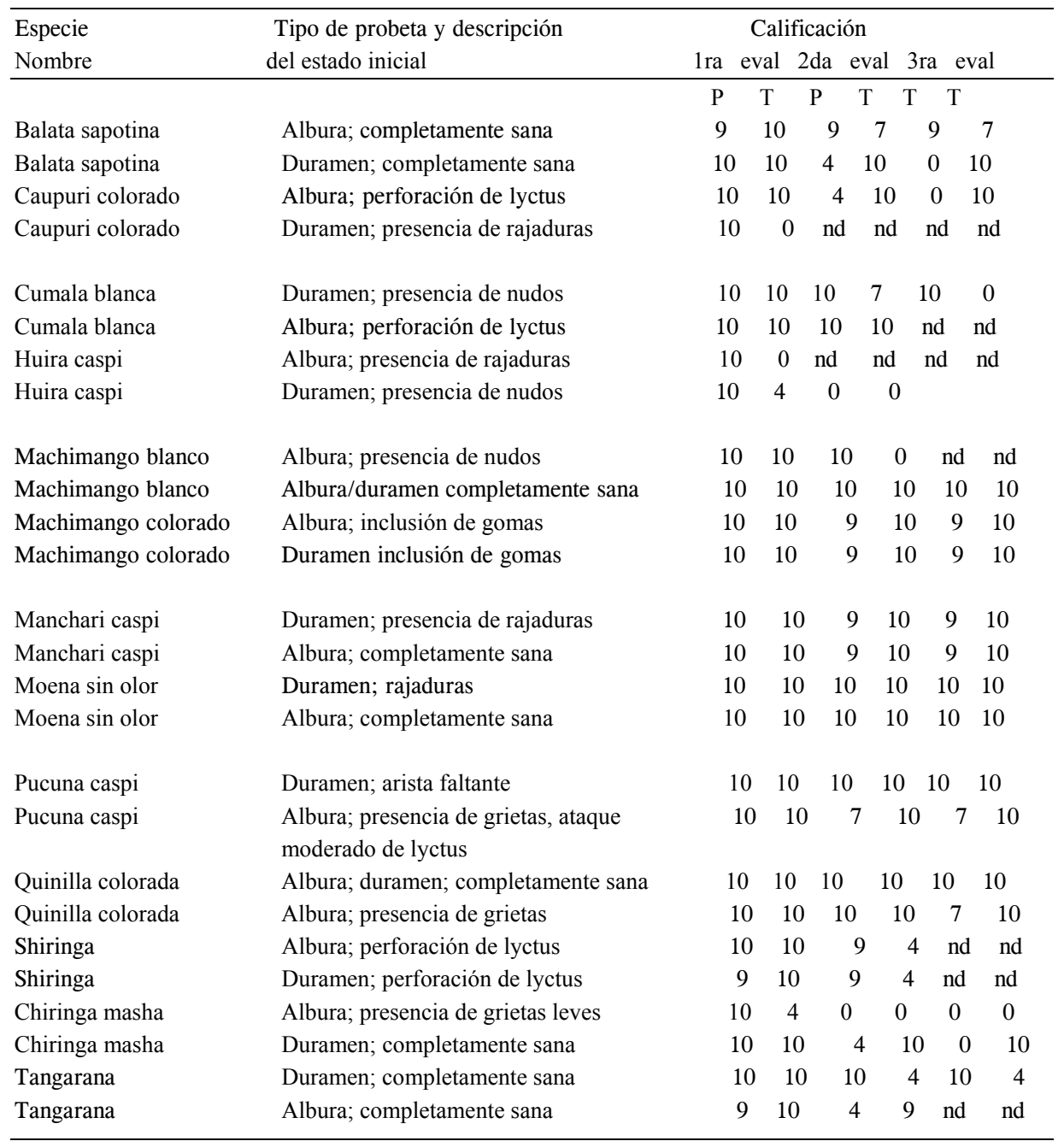

* $\quad$ Pudrición

** Termes 


\section{BIBLIOGRAFIA}

AMERICAN WOOD PRESERVER'S ASSOCIATTION. 1985. Standard M-7. Standard method for evaluation wood preserver's with plakes.

CARTWRIGHT, D.R. 1960. "Decay of timber and its preventio. Chemical Publishing. Brookling, N.Y.

GONZALES, V.R. 1974. Preservación de la Madera. Convenio UNALM-MEN. Lima. 72 pp.

HUNT, G.M. y GARRAT, G.A. 1962. Preservación de la madera Salvat Editores. Barcelona.

PADT-REFORT. 1981. Estudio integral de la madera para construcción (segunda fase). Normas y métodos para ensayos tecnológicos. Junta del Acuerdo de Cartagen. Lima. 